\title{
SOME NEW EXAMPLES OF COMPACT INHOMOGENEOUS HYPERCOMPLEX MANIFOLDS
}

\author{
Charles P. Boyer, Krzysztof Galicki, and Benjamin M. Mann
}

\begin{abstract}
We announce the construction of new families of compact, irreducible, inhomogeneous, hypercomplex manifolds which are not locally conformally hyperkähler. We obtain, for all $n>2$ and all $n$-tuples of non-zero real numbers $\mathbf{p}=\left(p_{1}, \ldots, p_{n}\right)$, a hypercomplex structure $\left\{\mathcal{I}^{a}(\mathbf{p})\right\}_{a=1,2,3}$ on the Stiefel manifold of 2-planes in $\mathbb{C}^{n}$. We determine the Lie algebra of infinitesimal hypercomplex automorphisms for each structure and show that among these structures there are uncountable families of pairwise inequivalent ones. Furthermore, these hypercomplex structures are inhomogeneous with the exception of the classical homogeneous spaces obtained when all the $p_{i}$ 's are equal. Finally, countably many of our examples admit discrete hypercomplex quotients by an action of the cyclic group of order $k$ and we analyze the topology of these non-simply connected examples. Full details and proofs appear in our paper [BGM3].
\end{abstract}

\section{Introduction}

Recently there has been much interest in quaternionic geometries [Sal2]. One such example is that of hypercomplex geometry. A smooth manifold $M$ is said to be a hypercomplex manifold if there exists three complex structures, $I, J$, and $K$ on $M$, satisfying the algebra of imaginary quaternions. Perhaps the simplest examples of hypercomplex manifolds are the hyperkähler manifolds where each of the three complex structures is actually Kähler. Until recently, examples of hypercomplex manifolds that are not hyperkähler were rare, the simplest ones being the Hopf manifolds $S^{4 n+3} \times S^{1}$ which are locally conformally hyperkähler. In [BGM2] we gave a new class of compact, locally conformally hyperkähler manifolds by replacing $S^{4 n+3}$ with any 3-Sasakian manifold. Hernandez [Her] found similar examples involving the quaternionic Heisenberg group. These nonhyperkähler examples, however, are not simply connected.

In dimension 4 all hypercomplex structures are locally conformally hyperkähler [Boy]; however, in higher dimensions this is no longer true. One class of hypercomplex manifolds that are not locally conformally hy-

1991 Mathematics Subject Classification. Primary 53C25.

Received August 3, 1994.

During the preparation of this work all three authors were supported by NSF grants. 
perkähler was first studied by physicists interested in supersymmetric $\sigma$ models and Spindel et. al. [SSTP] classified compact Lie groups which admit hypercomplex structures. This is the generalization to the hypercomplex category of the classic work of Samelson and Wang on the classification of compact Lie groups [Sam] and homogeneous spaces [W] admitting complex structures, respectively. Using different methods, Joyce [Joy1] later recovered this [SSTP] classification and developed a theory of homogeneous hypercomplex manifolds which generalizes Wang's [W] result in the complex case. However, inhomogeneous examples have been harder to find. With this background we announce the explicit construction of new classes of compact, irreducible, hypercomplex manifolds which are not locally conformally hyperkähler and almost all of which are inhomogeneous. The first and most basic class occurs on complex Stiefel manifolds and thus these examples are simply connected.

Theorem A. Let $n>2$ and $\mathbf{p}=\left(p_{1}, \ldots, p_{n}\right) \in\left(\mathbb{R}^{*}\right)^{n}$ be an $n$-tuple of non-zero real numbers. For each such $\mathbf{p}$ there is a compact hypercomplex manifold $\left(\mathcal{N}(\mathbf{p}), \mathcal{I}^{a}(\mathbf{p})\right)$, where $\mathcal{N}(\mathbf{p})$ is diffeomorphic to $\mathbb{V}_{n, 2}^{\mathbb{C}}$, the Stiefel manifold of 2-frames in $\mathbb{C}^{n}$. Moreover, $\mathcal{N}(\mathbf{p})$ is not locally conformally hyperkähler.

Permuting the coordinates of $\mathbf{p}$ or changing their signs in Theorem A yields the same hypercomplex structure. Thus, we assume that $\mathbf{p}$ is an element in the positive cone $C_{n}=\left\{\mathbf{p} \in \mathbb{R}^{n} \mid 0<p_{1} \leq p_{2} \leq \cdots \leq p_{n}\right\}$. However, we prove

Theorem B. If $\mathbf{p}$ and $\mathbf{q}$ are both commensurable sequences in the positive cone $C_{n}$ then the hypercomplex manifolds $\mathcal{N}(\mathbf{p})$ and $\mathcal{N}(\mathbf{q})$ are hypercomplex equivalent if and only if $\mathbf{p}=\mathbf{q}$. Here $\mathbf{p}$ is said to be commensurable if each of the ratios $\frac{p_{i}}{p_{j}}$ is a rational number. Furthermore, the manifold $\mathcal{N}(\mathbf{p})$ is hypercomplex homogeneous if and only if $\mathbf{p}=\lambda(1, \ldots, 1)$ for some $\lambda \in \mathbb{R}^{*}$.

While the one parameter family of distinct $U(n)$-homogeneous hypercomplex structures on $\mathbb{V}_{n, 2}^{\mathbb{C}}$ given in Theorem B was known [Joy1], the remaining inhomogeneous hypercomplex structures on $\mathbb{V}_{n, 2}^{\mathbb{C}}$ are new. They are analogous to the inhomogeneous complex structures found by Griffiths $[\mathrm{Gr}]$ in the versal deformation space of homogeneous complex structures.

Our next result shows that the connected component of the group of hypercomplex automorphisms of $\mathcal{N}(\mathbf{p})$ depends only on the number of equalities among the components of $\mathbf{p}$. More precisely, we rewrite $\mathbf{p}=$ $\left(p_{1}^{m_{1}}, \cdots, p_{k}^{m_{k}}\right)$, where the component $p_{i}$ occurs $m_{i}$ times in $\mathbf{p}$. The value 
$m_{i}$ is called the multiplicity of $p_{i}$ and the positive integer $k=k(\mathbf{p})$ counts the number of distinct values taken by the $p_{i}$ 's. We prove

Theorem C. For all $\mathbf{p} \in\left(\mathbb{R}^{*}\right)^{n}$ the Lie algebra $\mathfrak{h}(\mathbf{p})$ of infinitesimal hypercomplex automorphisms of $\mathcal{N}(\mathbf{p})$ is isomorphic to $\bigoplus_{i=1}^{k} \mathfrak{u}\left(m_{i}\right)$. Hence, the connected component of the group of hypercomplex automorphisms of $\mathcal{N}(\mathbf{p})$ is $\prod_{i=1}^{k} U\left(m_{i}\right)$. In particular, there exists a natural hyperhermitian metric $h(\mathbf{p})$ on $\mathcal{N}(\mathbf{p})$ such that every infinitesimal automorphism is an infinitesimal isometry with respect to $h(\mathbf{p})$.

Notice that Theorem $\mathrm{C}$ implies that the set of multiplicities in $\mathbf{p}$ is an invariant of the hypercomplex structure even when $\mathbf{p}$ is not a commensurable sequence and that if $\mathbf{p} \neq \lambda(1, \ldots, 1)$ then $\mathfrak{h}(\mathbf{p})$ is strictly smaller than the Lie algebra of infinitesimal hypercomplex automorphisms of the classical homogeneous structure on $\mathbb{V}_{n, 2}^{\mathbb{C}}$.

We now explain how these hypercomplex structures on $\mathbb{V}_{n, 2}^{\mathbb{C}}$ are related to some non-simply connected examples. To do so we first need to establish the following notation:

Definition D. A commensurable sequence $\mathbf{p} \in C_{n}$ is called basic if all the coordinates are integers and the greatest common divisor of all the coordinates is one. A basic sequence is said to be coprime if the coordinates are pairwise relatively prime. If $\mathbf{p}$ is an integer multiple of a basic sequence and if the triples $\left(p_{i}, p_{j}, k\right)$ have no common factor for all $1 \leq i<j \leq n$ then $\mathbf{p}$ is called $k$-coprime.

In [BGM2] we showed that for all coprime sequences $\mathbf{p}$ there is a 3 Sasakian manifold $\mathcal{S}(\mathbf{p})$ such that the product $\mathcal{S}(\mathbf{p}) \times S^{1}$ is a hypercomplex manifold. As mentioned above, these examples should be thought of as generalizations of Hopf manifolds as a 3-Sasakian manifold should be thought of as a generalization of the $(4 n+3)$-sphere. Moreover, there is a principal fibration $S^{1} \longrightarrow \mathcal{N}(\mathbf{p}) \longrightarrow \mathcal{S}(\mathbf{p})$. Thus, both $\mathcal{N}(\mathbf{p})$ and $\mathcal{S}(\mathbf{p}) \times S^{1}$ are hypercomplex manifolds which fibre over $\mathcal{S}(\mathbf{p})$ with circle fibres. The following theorem shows that these two spaces are the two extremes of new families of examples as their fundamental groups are 0 and $\mathbb{Z}$, respectively.

Theorem E. Let $\mathbf{p}$ be either coprime or $k$-coprime. There there is a compact hypercomplex manifold $\mathcal{H}(\mathbf{p}, k)$ with universal cover

$$
\rho_{k}: \mathcal{N}(\mathbf{p}) \longrightarrow \mathcal{H}(\mathbf{p}, k)
$$

such that $\pi_{1}(\mathcal{H}(\mathbf{p}, k)) \cong \mathbb{Z}_{k}$ and $\rho_{k}$ is a hypercomplex map. Moreover, $\mathcal{H}(\mathbf{p}, k)$ is never locally conformally hyperkähler and is hypercomplex homogeneous if and only if $\mathbf{p}=(p, p, \ldots, p)$. 
Theorems B,C, and E immediately imply

Corollary F. Let $\mathbf{p}$ be either coprime or $k$-coprime and $\mathbf{q}$ be either coprime or l-coprime. Then $\mathcal{H}(\mathbf{p}, k)$ is hypercomplex equivalent to $\mathcal{H}(\mathbf{q}, l)$ if and only if $\mathbf{p}=\mathbf{q}$ and $k=l$. Furthermore, the Lie algebra of infinitesimal hypercomplex automorphisms of $\mathcal{H}(\mathbf{p}, k)$ is the Lie algebra $\mathfrak{h}(\mathbf{p})$ given in Theorem $C$.

Remark. If $\mathbf{p}$ is commensurable but neither coprime nor $k$-coprime it is still possible to construct $\mathcal{H}(\mathbf{p}, k)$ and obtain a hypercomplex orbifold. However, if $\mathbf{p}$ is not commensurable then $\mathcal{H}(\mathbf{p}, k)$ is non-Hausdorff. While the topology of $\mathcal{N}(\mathbf{p})$ is well-known and independent of $\mathbf{p}$ we have

Theorem G. Let $\mathbf{p}$ be coprime and $k$ a positive integer. Then, as graded rings,

$$
H^{*}(\mathcal{H}(\mathbf{p}, k), \mathbb{Z}) \cong\left(\frac{\mathbb{Z}_{k}\left[x_{2}\right]}{\left[x_{2}^{n}=0\right]} \otimes E\left[y_{2 n-3}, z_{2 n-1}\right]\right) / \mathcal{R}(\mathcal{H}(\mathbf{p}, k))
$$

where the subscripts on $x_{2}, y_{2 n-3}$ and $z_{2 n-1}$ denote the cohomological dimension of each generator. The relations $\mathcal{R}(\mathcal{H}(\mathbf{p}, k))$ are given by

$$
d(\mathbf{p}, k) x_{2}^{n-1}=d(\mathbf{p}, k) x_{2} y_{2 n-3}=0
$$

and

$$
x_{2}^{2} y_{2 n-3}=x_{2}^{n-1} z_{2 n-1}=x_{2} y_{2 n-3} z_{2 n-1}=0 .
$$

Here $\sigma_{n-1}(\mathbf{p})$ is the $(n-1)^{\text {st }}$ elementary symmetric polynomial in the coordinates of $\mathbf{p}$ and $d(\mathbf{p}, k)=\operatorname{gcd}\left(\sigma_{n-1}(\mathbf{p}), k\right)$. The convention here is that $d(\mathbf{p}, 0)=\sigma_{n-1}(\mathbf{p})$.

Note that the topology of $\mathcal{H}(\mathbf{p}, k)$ depends on both $k$ and $\mathbf{p}$. For example,

Corollary H. For all coprime $\mathbf{p}, n \geq 3$, and $k>1$ there is one cohomological invariant of $\mathcal{H}(\mathbf{p}, k)$ that depends on $\mathbf{p}$; namely, the integer $d(\mathbf{p}, k)$ which is the order of the torsion subgroups of the $2 n-2$ and $2 n-1$ integral cohomology groups of $\mathcal{H}(\mathbf{p}, k)$. 


\section{Outline of proofs}

Our constructions were motivated by results in 3-Sasakian geometry in that we showed that any circle bundle over a 3-Sasakian manifold admits a natural almost hypercomplex structure and that this structure is integrable under some additional compatibility conditions. Our $\mathcal{N}(\mathbf{p})$ examples for coprime sequences $\mathbf{p}$ arose exactly this way. However, it became apparent that one could work on the $\mathcal{N}(\mathbf{p})$ level directly and drop the coprime and commensurable assumptions on $\mathbf{p}$. We now outline these ideas and refer to our paper [BGM3] for complete details and proofs.

Recall that an almost contact structure on a differentiable manifolds $M$ is a triple $(\Phi, \xi, \eta)$, where $\Phi$ is a tensor field of type $(1,1)$, $\xi$ is a vector field, and $\eta$ is a 1-form, which satisfy

$$
\eta(\xi)=1 \quad \text { and } \quad \Phi \circ \Phi=-\mathbb{I}+\xi \otimes \eta,
$$

where $\mathbb{I}$ is the identity endomorphism on $T M$. The following definition is due to Kuo $[\mathrm{Ku}]$.

Definition I. An almost contact 3-structure on $\mathcal{S}$ consists of 3 almost contact structures $\left(\Phi^{a}, \xi^{a}, \eta^{a}\right)_{a=1}^{3}$ which satisfy

$$
\begin{aligned}
\eta^{a}\left(\xi^{b}\right) & =\delta^{a b}, \\
\Phi^{a} \xi^{b} & =-\epsilon^{a b c} \xi^{c}, \\
\Phi^{a} \circ \Phi^{b}-\xi^{a} \otimes \eta^{b} & =-\epsilon^{a b c} \Phi^{c}-\delta^{a b} \mathbb{I} .
\end{aligned}
$$

Furthermore, if $g$ is an associated Riemannian metric on $\mathcal{S}$; that is, the metric that satisfies the conditions $g\left(X, \xi^{a}\right)=\eta^{a}(X)$ and $g\left(\Phi^{a} X, \Phi^{a} Y\right)=$ $g(X, Y)-\eta^{a}(X) \eta^{a}(Y)$, then we say that $\mathcal{S}$ is a 3 -Sasakian manifold if the vector fields $\xi^{a}$ are unit length Killing vector fields with respect to $g$ and

$$
\Phi^{a} X=\nabla_{X} \xi^{a} \quad \text { and } \quad\left(\nabla_{X} \Phi^{a}\right)(Y)=\eta^{a}(Y) X-g(X, Y) \xi^{a} .
$$

Next, let $\pi: P \rightarrow \mathcal{S}$ be a circle bundle over $\mathcal{S}$ and let $\hat{g}$ be a Riemannian metric on $P$ such that $\pi:(P, \hat{g}) \rightarrow(\mathcal{S}, g)$ is a Riemannian submersion. Let $\mathcal{V}_{1}$ denote the vertical subbundle of the tangent bundle $T P$ to $P$. Any nowhere vanishing smooth section $\Xi$ of $\mathcal{V}_{1}$ generates the $S^{1}$ action on $P$. The almost contact 3 -structure on $\mathcal{S}$ allows us to define an almost hypercomplex structure on $P$ as follows. The metric $\hat{g}$ on $P$ splits the tangent bundle $T P$ as $T P \simeq \hat{\mathcal{H}} \oplus \mathcal{V}_{1}$ and $\pi_{*}$ induces an isometry between the horizontal vector space $\hat{\mathcal{H}}_{p}$ at a point $p \in P$ and the tangent space $T_{\pi(p)} \mathcal{S}$. For any vector field $X$ on $\mathcal{S}$, we denote by $\hat{X}$ its horizontal lift to $P$, that is, $\hat{X}$ is the unique basic vector field that is $\pi$-related to $X$. In particular, the three vector fields $\hat{\xi}^{a}$ generate a subbundle $\hat{\mathcal{V}}_{3}$ of $\hat{\mathcal{H}}$ 
that is isometric at every point to the bundle $\mathcal{V}_{3}$ on $\mathcal{S}$. Let $\tilde{\mathcal{H}}$ denote the orthogonal complement to $\hat{\mathcal{V}}_{3}$ in $\hat{\mathcal{H}}$, so that we have the further splitting $T P \simeq \hat{\mathcal{H}} \oplus \mathcal{V}_{1} \simeq \tilde{\mathcal{H}} \oplus \hat{\mathcal{V}}_{3} \oplus \mathcal{V}_{1}$. Since the $\Phi^{a}$ 's are sections of End $\mathcal{H} \oplus$ End $\mathcal{V}_{3}$ on $\mathcal{S}$ they lift to sections $\hat{\Phi}^{a}$ of End $\tilde{\mathcal{H}} \oplus$ End $\hat{\mathcal{V}}_{3}$ on $P$ defined on basic vector fields by $\hat{\Phi}^{a} \hat{X}=\widehat{\Phi^{a} X}$ and extended to arbitrary sections of End $\tilde{\mathcal{H}} \oplus$ End $\hat{\mathcal{V}}_{3}$ by linearity. Hence, we can define endomorphisms $\mathcal{I}^{a}$ on $T P$ by

$$
\mathcal{I}^{a} X=-\hat{\Phi}^{a} X+\pi^{*} \eta^{a}(X) \Xi \text { and } \mathcal{I}^{a} \Xi=-\hat{\xi}^{a},
$$

where $X$ is any horizontal vector field on $P$. One easily sees that this defines an almost hypercomplex structure on $P$ which is, in general, not integrable. We shall denote by $H(\mathcal{S})$ this circle bundle with its almost hypercomplex structure.

Theorem J. Let $H(\mathcal{S})$ be a circle bundle over a 3-Sasakian manifold $\mathcal{S}$, and let $\Xi$ be a nowhere vanishing vertical vector field on $H(\mathcal{S})$ which generates the circle action. We call the pair $(H(\mathcal{S}), \Xi)$ a framed circle bundle on $\mathcal{S}$. The almost hypercomplex structure given above is integrable if and only if the horizontal subbundle $\hat{\mathcal{H}}$ defines a $\mathfrak{u}(1)$ connection with curvature 2-form $\omega$ and the following three conditions hold:

(i) $\omega\left(\hat{\xi}^{a}, \hat{\xi}^{b}\right)=0$ for any $a, b=1,2,3$.

(ii) $\omega\left(\hat{\xi}^{a}, \tilde{X}\right)=0$ for all $a=1,2,3$ and any section $\tilde{X}$ of $\tilde{\mathcal{H}}$.

(iii) $\omega\left(\hat{\Phi}^{a} \tilde{X}, \hat{\Phi}^{a} \tilde{Y}\right)=\omega(\tilde{X}, \tilde{Y})$ for all $a=1,2,3$ and for any sections $\tilde{X}, \tilde{Y}$ of $\tilde{\mathcal{H}}$.

Theorem $\mathrm{J}$ can now be shown to apply to the framed circle bundle $\mathcal{N}(\mathbf{p}) \rightarrow \mathcal{S}(\mathbf{p})$ constructed in [BGM1, BGM2]. Moreover, $\mathcal{N}(\mathbf{p})$ is naturally defined for all $\mathbf{p} \in C_{n}$ and the analysis in Theorem $\mathrm{J}$ directly generalizes to prove Theorem A.

When the compatibility conditions of Theorem J are satisfied, a beautiful picture emerges, namely there is a commutative diagram of foliations:

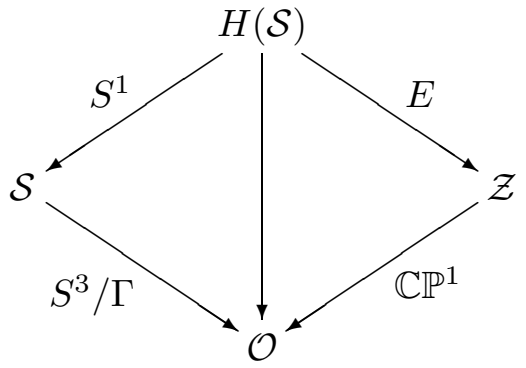

where $\mathcal{O}$ is a quaternionic Kähler orbifold and $\mathcal{Z}$ is its twistor space. Now $\mathcal{Z}$ is actually a projective algebraic variety with a Kähler-Einstein metric of positive scalar curvature, and $E$ is an elliptic curve. Furthermore, the leaves of the vertical foliation in diagram 2.5 are Hopf surfaces $S^{1} \times S^{3} / \Gamma$. 
Theorems B and C are proved by a detailed investigation of the hypercomplex geometry of the $\mathcal{N}(\mathbf{p})$ manifolds. Assuming that $\mathbf{p}$ is commensurable we first show that any hypercomplex equivalence $F: \mathcal{N}(\mathbf{p}) \rightarrow \mathcal{N}(\mathbf{q})$ would have to induce orbifold diffeomorphisms $F_{1}, F_{2}, F_{4}$ such that the following diagram commutes:

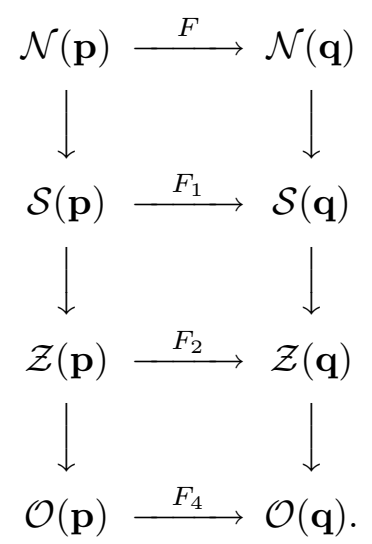

Theorem B then follows from a careful analysis of the holonomy groups of the leaves of the vertical foliation of diagram 2.5.

The proof of Theorem $\mathrm{C}$ entails the interplay between our hypercomplex Stiefel manifolds $\mathcal{N}(\mathbf{p})$ and equivalent models using Joyce's [Joy2] hypercomplex quotient procedure. This enables us to exhibit an invariance under scaling in $\mathbf{p}$ of the Lie algebra of the infinitesimal hypercomplex automorphisms $\mathfrak{h}(\mathbf{p})$ of $\mathcal{N}(\mathbf{p})$. This scale invariance in turn allows us to prove that any infinitesimal hypercomplex automorphism must also be an infinitesimal isometry with respect to a suitable metric. Then $\mathfrak{h}(\mathbf{p})$ can be calculated explicitly using standard results about isometric embeddings in $\mathbb{R}^{l}$.

Finally, Theorem E follows from Theorem A and a covering space argument while Theorem $\mathrm{G}$ follows from a standard Gysin sequence calculation and Theorem E of [BGM2].

\section{Acknowledgments}

We would like to thank Dr. H. Hernandez and Dr. T. Nitta for interesting remarks and discussions. The second author would like to thank Professor F. Hirzebruch and the Max-Planck Institute for support and hospitality. 


\section{References}

[Boy] C.P. Boyer, A note on hyperhermitian four-manifolds, Proc. AMS 102(1) (1988), 157-164.

[BGM1] C.P. Boyer K. Galicki and B.M. Mann, Quaternionic reduction and Einstein manifolds, Commun. Anal. Geom. 1(2) (1993), 229-279.

[BGM2] C.P. Boyer K. Galicki and B.M. Mann, The geometry and topology of 3Sasakian Manifolds, J. reine angew. Math. 455 (1994), 183-220.

[BGM3] C.P. Boyer K. Galicki and B.M. Mann, Hypercomplex structures on Stiefel manifolds, UNM preprint, 1994.

[Gr] P.A. Griffiths, Some geometric and analytic properties of homogeneous complex manifolds, parts I and II, Acta Math. 110 (1963), 115-208.

[Her] G. Hernandez, On hyper f-structures, Dissertation, Univ. of New Mexico, 1994.

[Joy1] D. Joyce, Compact hypercomplex and quaternionic manifolds, J. Diff. Geom. 35(3) (1992), 743-762.

[Joy2] D. Joyce, The hypercomplex quotient an the quaternionic quotient, Math. Ann. 290 (1991), 323-340.

[Ku] Y.-Y. Kuo, On almost contact 3-structure, Tôhoku Math. J. 22 (1970), 325332.

[Sal1] S. Salamon, Quaternionic Kähler manifolds, Invent. Math. 67 (1982), 143-171.

[Sal2] S. Salamon, Differential geometry of quaternionic manifolds, Ann. Scient. Ec. Norm. Sup. 19 (1986), 31-55.

[Sam] H. Samelson, A class of complex analytic manifolds, Portugal. Math. 12 (1953), 129-132.

[SSTP] Ph. Spindel, A. Sevrin, W. Troost, and A. Van Proeyen, Extended supersymmetric $\sigma$-models on group manifolds, Nucl. Phys. B308 (1988), 662-698.

[W] H.C. Wang, Closed manifolds with homogeneous complex structures, Am. J. Math. 76 (1954), 1-32.

Department of Mathematics and Statistics, University of New Mexico, Albuquerque, NM 87131

E-mail address: cboyer@math.unm.edu, galicki@math.unm.edu, mann@math.unm.edu 